\title{
A NEW FIXED POINT THEOREM AND ITS APPLICATION
}

\author{
BY W. V. PETRYSHYN ${ }^{1}$ \\ Communicated by Philip Hartman, August 9, 1971
}

Introduction. The purpose of this note is two-fold. In $\S 1$ we present a general fixed point theorem (Theorem 1 below) for 1-set-contractions and 1-ball-contractions defined on closures of bounded open subsets of Banach spaces. In $\$ 2$ we indicate briefly how Theorem 1 is used to deduce a number of known, as well as some new, fixed point theorems for various special classes of mappings which recently have been extensively studied and which are shown to be either 1-set-contractive or 1-ball-contractive. Complete proofs and detailed discussion of the results presented in this note will be given in [14].

1. A fixed point theorem. Let $X$ be a real Banach space, $D$ a subset of $X$ with $\bar{D}$ denoting its closure and $\partial D$ its boundary, $T$ a bounded continuous mapping of $\bar{D}$ into $X$. Following Kuratowski we say that $T$ is $k$-setcontractive if $\gamma(T(A)) \leqq k \gamma(A)$ for each bounded $A \subset \bar{D}$ and some constant $k \geqq 0$, where $\gamma(A)$ is the set-measure of noncompactness of $A$ given by $\inf \{r>0 \mid A$ can be covered by a finite number of sets of diameter $\leqq d\}$.

An important example of a $k$-set-contraction, $k<1$, is a mapping $T=S+C$ with $S: \bar{D} \rightarrow X$ strictly contractive (i.e., $\|S x-S y\| \leqq k\|x-y\|$ for $s, y \in \bar{D}, k<1)$ and $C: \bar{D} \rightarrow X$ compact. In [16] Sadovsky introduced a related class of mappings to which we refer here as ball-condensing, i.e., $T: \bar{D} \rightarrow X$ is such that $\chi(T(A))<\chi(A)$ for each bounded $A \subset \bar{D}$, where $\chi(A)$ is the ball-measure of noncompactness of $A$ given by

$$
\inf \{r>0 \mid A \text { can be covered by }
$$

a finite number of balls with centers in $X$ and radius $r$.

In analogy with $k$-set-contractions, we say that $T: \bar{D} \rightarrow X$ is $k$-ballcontractive if $\chi(T(A)) \leqq k \chi(A)$ for each bounded $A \subset \bar{D}$ and some $k \geqq 0$. The two classes of mappings, $k$-set-contractions and $k$-ball-contractions, are in fact different since they are defined in terms of measures $\gamma$ and $\chi$ which are known to be different although they have a great deal in common. It follows that a $k$-set-contraction with $k<1$ is set-condensing and that a set-condensing map is 1 -set-contractive, but the reverse implications

AMS 1969 subject classifications. Primary 4785, 4780; Secondary 5485.

Key words and phrases. Fixed point theorems, topological degree, i-set-contractions, 1-ball-contractions, mappings of contractive and semicontractive types.

${ }_{1}$ Partially supported by NSF GP-20228 and in part by the Research Council of Rutgers University. 
do not hold [11]. The same is true for mappings defined in terms of ballmeasures.

The main result of this section is the following new fixed point theorem.

THEOREM 1. Let $D$ be a bounded open subset of $X$ and T either a 1-setcontractive or a 1-ball-contractive mapping of $\bar{D}$ into $X$ for which the following holds:

(a) There exists $x_{0} \in D$ such that if Tx $-x_{0}=\alpha\left(x-x_{0}\right)$ holds for some $x$ in $\partial D$, then $\alpha \leqq 1$.

(b) If $\left\{x_{n}\right\}$ is any sequence in $\bar{D}$ such that $x_{n}-T\left(x_{n}\right) \rightarrow 0$ as $n \rightarrow \infty$, then there exists $x^{\prime} \in \bar{D}$ such that $x^{\prime}-T\left(x^{\prime}\right)=0$.

Then, in either case, Thas a fixed point in $\bar{D}$.

Note that (a) is implied by either of the assumptions:

$$
\begin{aligned}
\|T x-x\|^{2} & \geqq\left\|T x-x_{0}\right\|^{2}-\left\|x-x_{0}\right\|^{2} \quad \text { or } \\
(T x, J x) & \leqq(x, J x) \quad \text { if } 0 \in D \quad(x \in \partial D),
\end{aligned}
$$

where $J$ is any duality mapping of $X$ into $2^{X^{*}}$. In case $D$ is also convex, (a) is implied by the assumption: $T(\partial D) \subset \bar{D}$. Condition (b) is certainly implied by the stronger hypothesis that the set $(I-T)(\bar{D})$ be closed. The latter hypothesis holds, in particular, when $T$ is set-condensing or ballcondensing. Other mappings $T$ for which (b) holds will be discussed in $\$ 2$. Note that the mild condition (b) is in fact a necessary condition for $T$ to have a fixed point in $\bar{D}$.

The proof of Theorem 1 is based on the following properties of the degree of the mapping $I-T$ which we define in a way which is similar to the approach used in [13] for a more restrictive class of mappings.

Proposition 1. If $D$ is a bounded open subset of $X$ and $T: \bar{D} \rightarrow X$ is either 1-set-contractive or 1-ball-contractive with $\|(I-T)(x)\| \geqq \delta>0$ for $x$ in $\partial D$, then $\operatorname{deg}(I-T, D, 0)$ given by

$$
\operatorname{deg}(I-T, D, 0)=\lim _{j} \operatorname{deg}\left(I-k_{j} T, D, 0\right)
$$

is well defined, where $\left\{k_{j}\right\}$ is a sequence in $(0,1)$ such that $k_{j} \rightarrow 1$ as $j \rightarrow \infty$. Furthermore, if $\operatorname{deg}(I-T, D, 0) \neq 0$ and $T$ satisfies condition (b) of Theorem 1, then $T$ has a fixed point in $\bar{D}$.

Proposition 2. If $D$ is a bounded open subset of $X$ and $F: \bar{D} \times[0,1] \rightarrow X$ is a continuous mapping such that either $\gamma(F(A \times[0,1])) \leqq \gamma(A)$ or $\chi(F(A \times[0,1])) \leqq \chi(A)$ for each $A \subset \bar{D}$ and $\|x-F(x, t)\| \geqq \delta>0$ for $x$ in $\partial D$ and $t$ in $[0,1]$, then $\operatorname{deg}(I-F(\cdot, t), D, 0)$ is well defined and independent of $t \in[0,1]$. Furthermore, if $\operatorname{deg}(I-F(\cdot, 0) D, 0) \neq 0$ and $F(\cdot, 1)$ satisfies condition (b) on $\bar{D}$, then $F(\cdot, 1)$ has a fixed point in $\bar{D}$. 
The proof of Propositions 1 and 2 is based on the definition and the properties of the topological degree for the translations of $k$-set-contraction with $k<1$ obtained by Nussbaum [10]. It is not hard to show (see [18]) that the approach of [10] is also applicable to $k$-ball-contractions as defined here and consequently the same definition and properties hold also for the latter class of mappings.

REMARK 1 . Since $T: \bar{D} \rightarrow X$ is compact if and only if $T$ is 0 -set-contractive (or 0-ball-contractive), the theorems of Schauder [17], Rothe [15], Altman [1] and others (see [14]) are very special cases of Theorem 1. Also, since each set-condensing (or ball-condensing) map is 1-set-contractive (or 1-ball-contractive) and satisfies condition (b) on $\bar{D}$ and since (a) follows from the condition $T(\partial D) \subset \bar{D}$ when $D$ is also convex, the fixed point theorems of Darbo [5], Sadovsky [16], Nussbaum [10], [11], Furi and Vignoli [6] and others (see [14]) are special cases of Theorem 1. For the case when $T$ is 1-set-contractive and $(I-T)(\bar{D})$ is closed, Theorem 1 has been obtained by the writer (see Theorem $7^{\prime}$ in Petryshyn [12]).

We add that Theorem 1 is a proper extension of Theorem $7^{\prime}$ in [12] for there are mappings (e.g., generalized contractions defined only on $\bar{D}$ ) for which condition (c) holds but for which $(I-T)(\bar{D})$ need not be closed; furthermore, there are mappings (e.g., semicontractive maps defined on all of $X$ ) which are 1-ball-contractive but need not be 1-set-contractive.

2. Applications. In this section we use Theorem 1 to deduce a number of fixed point theorems (some known and some new) for special classes of mappings which were obtained earlier by other authors using different methods and, in some cases, under conditions on $T$ and/or $D$ which are more stringent than those imposed here. Our object is to illustrate the generality and the unifying property of Theorem 1 . We concentrate in this note on the class of contractive mappings with perturbations.

The first consequence of Theorem 1 is the following corollary which is new in the sense that unlike all other authors (e.g., [9], [19], [4]) we do not require $D$ to be convex.

Corollary 1. If $D$ is a bounded open subset of $X, S: \bar{D} \rightarrow X$ strictly contractive, $C: \bar{D} \rightarrow X$ compact, and $T=S+C$ satisfies condition (a) of Theorem 1 , then $T$ has a fixed point in $\bar{D}$.

REMARK 2. Special cases of Corollary 1 have been obtained earlier by a number of authors (see [14] for historical remarks concerning various contributions).

Before we state our next corollary, we recall that $P: \bar{D} \rightarrow X$ is completely continuous if $\left\{x_{n}\right\}$ is any sequence in $\bar{D}$ such that $x_{n} \rightarrow x_{0} \in \bar{D}$ weakly, then $P\left(x_{n}\right) \rightarrow P\left(x_{0}\right)$ strongly; $P$ is demiclosed if $x_{n} \rightarrow x_{0}$ in $\bar{D}$ weakly and $P\left(x_{n}\right) \rightarrow f$ strongly, then $P\left(x_{0}\right)=f ; P$ is nonexpansive if $\|P x-P y\|$ 
$\leqq\|x-y\|$ for $x, y \in \bar{D}$. In view of Theorem 2 of Browder [4], Theorem 1 yields the following corollary.

COROLlary 2. If $X$ is uniformly convex, $D$ a bounded open convex subset of $X, S: \bar{D} \rightarrow X$ nonexpansive, $C: \bar{D} \rightarrow X$ completely continuous, and $T=S+C$ satisfies condition (a) of Theorem 1, then Thas a fixed point in $\bar{D}$.

Remark 3. In case $C=0$ and $T(\bar{D}) \subset \bar{D}$, Corollary 2 has been first established independently by Browder [3], Kirk [8], and Göhde [7]. In case $C \neq 0$, very special cases of Corollary 2 have been obtained by a number of authors (see [14] for historical comments). Subsequently, Corollary 2 has been obtained by Browder as a special case of more general results obtained in [3] by means of a rather complicated theory of initial value problems for differential equations in $X$. We add in passing that the fixed point theorems in [4] will also be shown in [14] to be special cases of Theorem 1 obtained under less restrictive conditions than those imposed in [4].

If in Corollary 2 we assume that $S$ is a generalized contraction in the sense of [2] (i.e., to each $x$ in $\bar{D}$ there exists $\alpha(x)<1$ such that $\|S x-S y\|$ $\leqq \alpha(x)\|x-y\|$ for $y$ in $\bar{D})$, then the condition on $X$ can be relaxed.

COROllary 3. If $X$ is reflexive, $D$ a bounded open convex subset of $X$, $C: \bar{D} \rightarrow X$ completely continuous, $S: \bar{D} \rightarrow X$ a generalized contraction, and $T=S+C$ such that

$$
S(x)+C(y) \in \bar{D} \quad \text { for } x, y \in D,
$$

then Thas a fixed point in $\bar{D}$.

Corollary 3 follows from Theorem 1 and a lemma which states that if $T=S+C$ satisfies the hypotheses of Corollary 3 , then $T$ satisfies condition (b) of Theorem 1. Note that since the set $(I-T)(\bar{D})$ need not be closed, Corollary 3 shows that Theorem 1 is indeed a proper generalization of Theorem $7^{\prime}$ in [12] even for 1-set-contractions.

In case $C=0$, Corollary 3 has been obtained in [2] from their results on mappings of "diminishing orbital diameters."

If in Corollary 3 we assume that $D=B(0, r)$, then condition $(\mathrm{K})$ can be replaced by a weaker requirement:

$$
S(x)+C(y) \in \bar{D} \text { for } x \text { in } \partial B \text { and } y \text { in } \bar{B} \text {. }
$$

Finally we add that if $S$ in Corollary 3 is uniformly strictly contractive on $\bar{D}$ relative to $X$ in the sense of Kirk, (i.e., $\mathrm{S}: X \rightarrow X$ is such that to each $x$ in $X$ there exists $\alpha(x)<1$ such that $\|S x-S y\| \leqq \alpha(x)\|x-y\|$ for $y$ in $\bar{D})$ then the following strengthened result holds. 
Corollary 4. If $X$ is reflexive, $D$ a bounded open convex subset of $X$, $C: \bar{D} \rightarrow X$ compact, $S: X \rightarrow X$ uniformly strictly contractive on $\bar{D}$ relative to $X$, and $T=S+C$ satisfies condition (a) of Theorem 1 , then $T$ has a fixed point in $\bar{D}$.

The application of Theorem 1 to several more general classes of mappings such as everywhere defined semicontractive mappings, mappings of semiconductive type defined on subsets of $X$, mappings of pseudocontractive type, lane mappings, and others will be discussed in [14].

\section{REFERENCES}

1. M. Altman, A fixed point theorem in Banach space, Bull. Acad. Polon. Sci. Cl. III. 5 (1957), 19-22. MR 19, 297.

2. L. P. Belluce and W. A. Kirk, Fixed-point theorems for certain classes of nonexpansive mappings, Proc. Amer. Math. Soc. 20 (1969), 141-146. MR 38 \# 1663.

3. F. E. Browder, Nonexpansive nonlinear operators in a Banach space, Proc. Nat. Acad. Sci. U.S.A. 54 (1965), 1041-1044. MR 32 \# 4574.

4. - Semicontractive and semiaccretive nonlinear mappings in Banach spaces, Bull. Amer. Math. Soc. 74 (1968), 660-665. MR. 37 \# 5742.

5. G. Darbo, Punti uniti in transformazioni a condominio non compatto, Rend. Sem. Mat. Univ. Padova 24 (1955), 84-92. MR 16 \#1140.

6. M. Furi and A. Vignoli, On $\alpha$-nonexpansive mappings and fixed points, Atti Accad. Naz. Lincei Rend. Cl. Sci. Fis. Mat. Natur. (8) 48 (1970), 195-198.

7. D. Göhde, Zum Prinzip der kontraktiven Abbildung, Math. Nachr. 30 (1965), 251-258. MR 32 \# 8129.

8. W. A. Kirk, A fixed point theorem for mappings which do not increase distances, Amer. Math. Monthly 72 (1965), 1004-1006. MR 32 \#6436.

9. M. A. Krasnosel'skiǐ, Two remarks on the method of successive approximations, Uspehi Mat. Nauk 10 (1955), no. 1 (63), 123-127. (Russian) MR 16, 833.

10. R. D. Nussbaum, The fixed point index and fixed point theorems for $k$-set-contractions, Ph.D. Thesis, University of Chicago, Chicago, Ill., 1969.

11. - The fixed point index for local condensing maps, Ann. Mat. Pura Appl. (to appear).

12. W. V. Petryshyn, Structure of the fixed points sets of $k$-set-contractions, Arch. Rational Mech. Anal. 40 (1971), 312-328.

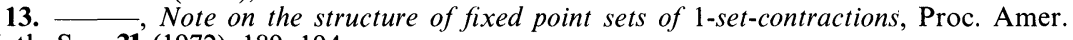
Math. Soc. 31 (1972), 189-194.

14. - Fixed point theorems for various classes of 1-set-contractive and 1-ballcontractive mappings in Banach spaces (in preparation).

15. E. Rothe, Zur Theorie der topologischen Ordnung und der Vektorfelder in Banachschen Räumen, Compositio Math. 5 (1937), 177-197.

16. B. N. Sadovskiǔ, On a fixed point principle, Funkcional. Anal. i Priložen. 1 (1967), no. 2, 74-76. (Russian) MR 35 \#2184.

17. J. Schauder, Der Fixpunktsatz in Funktionalräumen, Studia Math. 2 (1930), 171-180.

18. J. R. L. Webb, Remarks on k-set-contractions, Bolletino U.M.I. 4 (1971), 614-629.

19. P. P. Zabreǐko and M. A. Krasnosel'skil̆, A method for producing new fixed point theorems, Dokl. Akad. Nauk SSSR 176 (1967), 1233-1235 = Soviet Math. Dokl. 8 (1967), 1297-1299. MR 36 \#3183.

Department of Mathematics, Rutgers University, New Brunswick, New Jersey 08903 